This paper reports a study into the dependence of efficient glycerate potassium production involving glycerin and potassium hydroxide solution on the process conditions. The concentration of potassium glycerate in the resulting product has been used as a parameter of the efficient glycerate potassium production process.

Glycerates of metals are applied to produce articles in the construction industry, electronics, medicine; they are employed as transesterification catalysts to obtain special fats for various purposes, as well as biodiesel fuel.

In order to derive potassium glycerate, heating was applied while agitating a mixture of glycerin and potassium hydroxide.

The analysis of potassium hydroxide was performed, in which the basic substance mass fraction was $85.5 \%$, the mass fraction of carbonate potassium $0.9 \%$. The p.a.-grade glycerin was applied in this work, whose basic substance mass fraction was $99.5 \%$.

The effect of the heating temperature of the reaction mixture on the concentration of potassium glycerate in the product has been determined. It has been shown that the rational heating temperature is $145^{\circ} \mathrm{C}$.

The dependence of potassium glycerate concentration in the resulting product on the following conditions of the process has been established: a change in the molar concentration of glycerin and a reaction mixture heating duration.

Such rational conditions for obtaining potassium glycerate have been defined as a molar concentration of glycerin of $60 \%$ and a heating duration of 4 hours. The experimentally established concentration of potassium glycerate in the product under these conditions was $75.77 \%$.

For potassium glycerate, the melting point $\left(69^{\circ} \mathrm{C}\right)$ and the mass fraction of moisture $(0.8 \%)$ have been determined.

The results of experimental studies would make it possible to obtain potassium glycerate directly at enterprises where the glycerates of metals are used, from available raw materials, under rational conditions. The defined conditions for obtaining potassium glycerate could make it possible to efficiently utilize material and energy resources

Keywords: potassium glycerate, potassium hydroxide, glycerin, reaction mixture, basic substance content
UDC 661.7

DOI: 10.15587/1729-4061.2021.231449

\section{ESTABLISHING RATIONAL CONDITIONS FOR OBTAINING POTASSIUM GLYCERATE}

\author{
Natalia Sytnik \\ Corresponding author \\ $\mathrm{PhD}$ * \\ E-mail:ntlsytnik@gmail.com \\ Ekaterina Kunitsia \\ $\mathrm{PhD}$
}

Department of Innovative Food and Restaurant Technologies Kharkiv Institute of Trade and Economics of Kyiv National University of Trade and Economics

O. Yarosh lane, 8, Kharkiv, Ukraine, 61045

Viktoria Mazaeva $\mathrm{PhD*}$

Anton Chernukha $\mathrm{PhD}$ **

Kostiantyn Ostapov

$\mathrm{PhD}$

Department of Fire Tactics and Rescue Operations***

Pavlo Borodych

$\mathrm{PhD}$, Associate Professor**

Valeri Mazurenko

$\mathrm{PhD}$, Associate Professor

Department of Organizational Entry of a Civil Defence Institute of Public Administration and Research in Civil Protection Rybalska str., 18, Kyiv, Ukraine, 01011

O leks andr Kovalov

$\mathrm{PhD}$, Associate Professor

Department of Organizational Entry of a Civil Defence Institute of Public Administration and Research in Civil Protection Rybalska str., 18, Kyiv, Ukraine, 01011

Victoria VeIma $\mathrm{PhD}$

Department of Chemistry of Natural Compounds and Nutriciology National University of Pharmacy Pushkinska str., 53, Kharkiv, Ukraine, 61002

Vitalii Kolokolov

Department of Physical Training***

*Department of Studies of Technology for Processing Oils and Fats Ukrainian Scientific Research Institute of Oils and Fats of the National Academy of Agricultural Sciences of Ukraine Dziuby ave., 2a, Kharkiv, Ukraine, 61019 **Department of Fire and Rescue Training*** $* * *$ National University of Civil Defence of Ukraine Chernyshevska str., 94, Kharkiv, Ukraine, 61023
Received date: 18.01 .2021 Accepted date: 16.03 .2021 Published date: 18.06 .2021
How to Cite: Sytnik, N., Kunitsia, E., Mazaeva, V., Chernukha, A., Ostapov, K., Borodych, P., Mazurenko, V., Kovalov, O., Velma, V., Kolokolov, V. (2021). Establishing rational conditions for obtaining potassium glycerate. Eastern-European Journal of Enterprise Technologies, 3 (6 (111)), 12-18. doi: https://doi.org/10.15587/1729-4061.2021.231449
1. Introduction

Glycerates of metals that are used in various industries are glycerin salts. The reactivity of glycerin is determined by the presence in the molecule of this compound of two primary and one secondary hydroxylic groups. Glycerin exhibits the properties of a weak tribasic acid, and, unlike monoatomic alcohols, can react with alkaline metal hydroxides to form 
glycerates. Glycerin also forms halogen esters, salts with heavy metal oxides, as well as other compounds.

Glycerates of alkaline and alkaline earth metals are obtained by the interaction of glycerin with metals, oxides, and hydroxides of metals. Glycerates are widely used. Thus, sodium glycerate is applied in the production of absolute alcohol due to hygroscopic properties. Plumbum glycerate is used as a catalyst when obtaining alkyd resins. Calcium glycerate is applied for the adsorption of carbon dioxide and water [1]. Zinc glycerate is used in medicine [2].

Copper glycerate is applied during chemical glass processing; it can be used in the manufacture of fluorescent and photoluminescent coatings, as well as other elements of optoelectronics [3].

Glycerates of alkaline metals (sodium, potassium, lithium) are employed in the process of obtaining biodiesel fuel from vegetable oils and the waste of oil processing [4]. Calcium glycerate [5] is also used for this purpose. These compounds are catalysts for the transesterification process, as a result of which biodiesel fuel is produced, which is the esters of fatty acids.

Thus, there is a task to produce these substances at enterprises that apply technologies involving their use. Under the conditions of an enterprise where periodic or continuous production schemes are in place, a significant volume of products is manufactured with continuous quality control over products. Therefore, it is important to ensure the smooth operation of equipment, to avoid defects, to rationally utilize material and energy resources, to reduce the cost of products, to manage production safety [6]. All these factors affect the quality and economic feasibility of production.

There are various methods and technologies for obtaining metal glycerates, which differ in the set of initial reagents, as well as the process parameters [1]. However, to implement technology, there is a need to define rational conditions under which the technology would be efficient and cost-effective.

Thus, it is a relevant task to undertake a study aimed at determining the rational parameters for reaction, the ratio of components of the reaction mixture for obtaining glycerates of metals. Given the prospects of using these compounds in the priority industrial sectors, in particular, to produce fuel of the new generation, it is necessary to devise an effective technology for obtaining the glycerates of alkaline metals.

\section{Literature review and problem statement}

The most common way to obtain alkaline metal glycerates involves treating glycerin with a concentrated aqueous solution of sodium or potassium hydroxide during heating. The reaction temperature should ensure effective evaporation of moisture, therefore, should be above $100{ }^{\circ} \mathrm{C}$ [1].

Paper [7] reports the results of studying the production of potassium glycerate to use it as a catalyst in the production of biodiesel fuel. It was established that rational conditions are the molar ratio of potassium hydroxide to glycerin of $2: 1$, a temperature of $130{ }^{\circ} \mathrm{C}$. It is shown that the yield of biodiesel with the resulting potassium glycerate corresponds to the yield of biodiesel when using a regular industrial catalyst of potassium methoxide. However, there are no data on the impact of the duration of the components' reaction on the yield of potassium glycerate and its concentration in the resulting product. The cited work used a significant excess of potassium hydroxide when obtaining a catalyst. Consequently, the resulting catalyst has a significant concentration of alkali, which could lead to the saponification of fats and fatty acids in the production of biodiesel, and, as a result, the loss of raw materials.

The authors of [8] reported data on the production of calcium glycerate. Calcium glycerate has been shown to be reused for at least five reaction cycles without loss of activity during biodiesel fuel production. However, there are no data on the rational conditions for obtaining such a catalyst, the impact of temperature, and the duration of reaction on the catalyst yield and its concentration in the resulting product.

Work [9] examined the synthesis of calcium diglycerate by the mechanochemical treatment of calcium oxide dispersed in glycerin. The resulting product was tested as a catalyst for the methanolysis of sunflower oil. However, there are no data on the dependence of the effectiveness of obtaining calcium diglycerate on the synthesis parameters.

Paper [10] investigated the production of calcium diglyceride by the interaction of calcium hydroxide with glycerin with varying time and reaction temperature. However, there are no data on the effect of the ratio of the components of the reaction mixture on the effectiveness of the process of calcium diglyceride synthesis.

The authors of [11] obtained sodium glycerate from glycerin, methanol, and sodium hydroxide. At the same time, the influence of parameters and ratios of reagents on the output of the final product and the concentration of the main substance in it is not specified.

Thus, the process to obtain metal glycerates is an important scientific and practical task. However, current studies fail to provide enough data on the simultaneous influence of the main parameters on the concentration of glycerates in the resulting product. This information is important for the effective process of synthesis, rational inclusion of the production of glycerates in the general manufacturing scheme for obtaining products with their use. In addition, there is no information on the concentration of the basic substance in products obtained using technologies described in current scientific papers. The basic substance concentration is one of the most important characteristics of the synthesized substances since it determines the rate of introduction, the effectiveness of the use of the substance, its physicochemical properties, the behavior in the mixture with other components. These data are necessary to ensure the proper quality of products [12-14].

Thus, an unresolved issue in this area is the study of the rational ratio of reagents during the production of alkaline metal glycerates, as well as the establishment of rational parameters for the process of reaction of the components of the reaction mixture. It is necessary to determine the basic substance concentration in the resulting product, which would make it possible to accurately assess the required rate of use of glycerate and effectively conduct the process under established conditions.

\section{The aim and objectives of the study}

The aim of this study was to establish rational conditions for obtaining potassium glycerate. That could make it possible to effectively implement the production of potassium glycerate. The application of research results would contribute to the rational utilization of material and energy 
resources, improve the profitability of enterprises in various industries through the production and possible sale of high-quality products - alkaline metal glycerates.

To achieve the set aim, the following tasks have been solved:

- to analyze the raw material for the synthesis of potassium glycerate - potassium hydroxide; to determine the basic substance mass fraction and the mass fraction of potassium carbonate;

- to investigate the effect of the heating temperature of the reaction mixture on the effectiveness of obtaining potassium glycerate;

- to experimentally determine the dependence of the concentration of potassium glycerate in the resulting product on the process conditions: a change in the molar concentration of glycerin in the reaction mixture and the duration of heating;

- to determine the qualitative indicators of the obtained potassium glycerate: the melting point and mass fraction of moisture.

\section{Materials and methods to study the dependence of the effectiveness of obtaining potassium glycerate on the conditions of reaction}

\section{1. The studied materials and equipment used in the} experiment study:

The following reagents and materials were used in this

- p.a.-grade glycerin; concentration, $99.5 \%$ (CAS Number 56-81-5);

- p.a.-grade potassium hydroxide (the basic substance mass fraction is not less than $85.0 \%$ ) (CAS Number 1310-58-3);

- pur.-grade butanol (the basic substance mass fraction is $99.7 \%$; the mass fraction of water is $0.05 \%$ ) (CAS Number 71-36-3);

- molecular sieve (CAS Number 1318-02-1);

- puriss.-grade acetic acid with a concentration of 99.8 \% (CAS Number 64-19-7);

- p.a.-grade phenol phthalein (the basic substance mass fraction is not less than 98.0\%) (CAS Number 77-09-85768-87-6);

- methyl orange (the basic substance mass fraction is not less than $98.0 \%$ ) (CAS Number 547-58-0).

\section{2. Potassium hydroxide analysis procedure}

The basic substance mass fraction and the mass fraction of carbonate potassium in potassium hydroxide were determined according to the standard procedure in line with GOST 24363. The permissible differences between the two parallel measurements did not exceed $0.5 \%$ for potassium hydroxide, for potassium carbonate $0.2 \%$.

\section{3. Procedure to obtain potassium glycerate}

Glycerin and potassium hydroxide solution with a concentration of $50 \%$ are placed in a heat-resistant flask, the mixture is thoroughly agitated with a glass stick. The flask is fixed on a tripod and installed in a glycerin bath with a magnetic stirrer, the predefined temperature is set using a thermostat, the flask is connected to a vacuum pump. Agitating the contents of the flask is carried out during the specified reaction time, while water evaporation occurs in a vacuum, after which a viscous crystalline mass - potassium glycerate - is obtained.
4. 4. Procedure for determining the basic substance concentration in potassium glycerate

The method involves a potentiometric titration of the weighted portion, dissolved in dehydration butanol, with a solution of acetic acid. The weighted portion $(5 \pm 0.5) \mathrm{g}$ is placed in a glass with a volume of $100 \mathrm{~cm}^{3}, 30 \mathrm{~cm}^{3}$ of butanol is added and then dissolved in a water bath with a reverse refrigerator. The glass is placed on the titrating table, an electrode and a magnet are immersed in the solution to agitate the solution. One enables a magnetic stirrer to titrate the analyzed product with a solution of acetic acid in butanol, adding it in portions from 0.1 to $1.0 \mathrm{~cm}^{3}$, to a decrease in the $\mathrm{pH}$ of the medium to a constant value.

The data acquired are used to build the integrated and differential titration curves. The equivalent volume of an acid solution of $0.1 \mathrm{~mol} / \mathrm{dm}^{3} V_{e}, \mathrm{~cm}^{3}$, used to titrate the weighted portion of the analyzed product, is determined graphically (projection on the abscissa axis of the inflection point of the integrated curve or the maximum point on the differential titration curve).

The concentration of alkali (potassium hydroxide and potassium glycerate) in the product, \%, was determined from the following formula:

$$
X=\frac{c \cdot K \cdot V_{e} \cdot M}{m}
$$

where $c$ is the concentration of acetic acid, $\mathrm{mmol} / \mathrm{cm}^{3} ; K$ is the correction factor to the titer of acetic acid; $V_{e}$ is the equivalent volume of acetic acid solution used on titration, $\mathrm{cm}^{3} ; M$ is the molecular weight of alkali, $\mathrm{mg} / \mathrm{mmol} ; m$ is the weight of the product batch, g.

4. 5. Procedure for determining the melting point and mass fraction of moisture in potassium glycerate

The melting point of potassium glycerate was determined by a thermomicromethod according to the standard procedure in line with GOST 18995.4. The analysis result was the arithmetic mean of the results from two parallel measurements, the absolute discrepancy between which did not exceed the permissible difference equal to $0.5^{\circ} \mathrm{C}$ for thermometers with a scale division of 0.1 and $0.2{ }^{\circ} \mathrm{C}$, and $1{ }^{\circ} \mathrm{C}$ for thermometers with a scale division of $0.5^{\circ} \mathrm{C}$. The absolute total error of determining is $\pm 0.3^{\circ} \mathrm{C}$ for thermometers with a scale division of 0.1 and $0.2{ }^{\circ} \mathrm{C}$, and $\pm 1{ }^{\circ} \mathrm{C}$ for thermometers with a scale division of $0.5^{\circ} \mathrm{C}$ at a confidence probability $P=0.95$.

The mass fraction of moisture in potassium glycerate was determined as follows. $(2 \pm 0.2) \mathrm{g}$ of substance is placed in a porcelain bowl. The bowl is placed in the dryer, with a temperature of $105{ }^{\circ} \mathrm{C}$, for 45 minutes. After that, the bowl is placed in an exicator for 15 minutes. After that, weighing with an accuracy of up to 0.001 is carried out. Thus, drying cycles are repeated until the mass becomes constant. The differences between parallel measurements did not exceed $0.2 \%$.

\subsection{Planning the experimental study and processing} the results

To plan our scientific research and to process the data obtained, a full second-order factor experiment was used involving the calculations in the software packages Microsoft Office Excel 2003 (USA) and Stat Thusft Statistica v6.0 (USA). The research was carried out with a double repetition. 
5. The results of studying the dependence of the effectiveness of glycerate potassium production on the processing conditions of the reaction mixture

5. 1. Establishing the basic substance mass fraction in potassium hydroxide

During the storage of potassium hydroxide, a certain amount of carbonate potassium is formed in it. Potassium hydroxide was analyzed for the purpose of accurate dosage for potassium glycerate production. The mass fraction of the main substance of $85.5 \%$ was established, as well as the mass fraction of carbonate potassium, $0.9 \%$, according to the standard procedure (chapter 4. 2). Solution of potassium hydroxide with a concentration of $50 \%$ for potassium glycerate production was prepared taking into consideration the basic substance mass fraction.

5. 2. Investigating the dependence of potassium glycerate concentration in the resulting product on the heating temperature of the reaction mixture

Glycerates of alkali metals form in the conditions of heating and evaporation of moisture from the reaction mass. We examined the influence of the heating temperature of reagents within $(100-160){ }^{\circ} \mathrm{C}$ on the effectiveness of potassium glycerate formation (the basic substance concentration in the resulting product). Potassium glycerate in our study was obtained according to the procedure given in chapter 4. 3. The basic substance concentration in the resulting product was determined by the procedure described in chapter 4. 4. The results of our research are shown in Fig. 1. the increase in the basic substance mass fraction is sharply reduced after a temperature of $145^{\circ} \mathrm{C}$. Further studies were carried out at a temperature of $145^{\circ} \mathrm{C}$.

5. 3. Investigating the dependence of potassium glycerate concentration in the resulting product on the conditions of product manufacturing

Thus, it turned out that the process of formation of potassium glycerate at a temperature of at least $145^{\circ} \mathrm{C}$ is effective. When obtaining alkali metal glycerates, an important technological parameter is the ratio of components. During the heating of aqueous solutions of glycerin, partial evaporation of glycerin occurs. Hence, it is advisable to use an excess of glycerin. In addition, potassium hydroxide, which can remain in the resulting mass without adding excess glycerin, can complicate the further use of potassium glycerate (for example, in the transesterification of fats, it could cause fat loss due to saponification).

The following conditions of potassium glycerate production were investigated: a change in the molar concentration of glycerin in the mixture and the duration of reaction of the reaction mixture. For our experimental research and to build a mathematical model, a full factor experiment of the second order was used: the number of factors is 2 , the number of experiments is 9 , the number of levels is 3 . Variation intervals of factors: $x_{1}$ - molar concentration of glycerin in the mixture: from 50 to $70 \% ; x_{2}$ - reaction duration of reaction mixture: from 2 to 6 hours. Table 1 gives a matrix of planning experimental studies with the actual values of factors, as well as the experimentally established values of the response function - the concentration of potassium glycerate in the resulting product. At all "experimental points", potassium glycerate was obtained according to the procedure described in chapter 4.3; the basic substance concentration was determined according to the procedure described in chapter 4.4 .

The result of treating experimental data using the software package Stat Soft Statistica v6.0 (USA) (chapter 4.6) is the derived mathematical model, which is the dependence of the concentration of potassium glycerate in the resulting product on the processing conditions of the reaction mixture.

In a normalized form, the regression dependence of the concentration of potassium glycerate in the resulting product on the processing conditions of the reaction mixture is as follows:
Fig. 1. Dependence of the mass fraction of potassium glycerate in the resulting product on the heating temperature of the mixture of reagents

The approximation equation is as follows:

$$
y=14.159 \cdot \ln x+4.5812,
$$

where $x$ is the heating temperature of the mixture of reagents, ${ }^{\circ} \mathrm{C} ; y$ is the concentration of potassium glycerate in the resulting product, $\%$.

The degree of reliability of approximation was $R^{2}=0.9429$.

At a temperature of $120{ }^{\circ} \mathrm{C}$, the mass fraction of potassium glycerate in the resulting product is $71.4 \%$; at a temperature of $145^{\circ} \mathrm{C}, 75.8 \%$; at a temperature of $160{ }^{\circ} \mathrm{C}, 76.2 \%$. That is, the difference in mass fractions at $120^{\circ} \mathrm{C}$ and $145^{\circ} \mathrm{C}$ is $4.4 \%$; at $145{ }^{\circ} \mathrm{C}$ and $160{ }^{\circ} \mathrm{C}-$ only $0.4 \%$. Consequently,

$$
y=71.02+10.64 \cdot x_{1}+17.29 \cdot x_{2}-12.48 \cdot x_{2}^{2} \text {. }
$$

In actual variables:

$$
y=-77.30+1.06 \cdot x_{1}+33.60 \cdot x_{2}-3.12 \cdot x_{2}^{2} .
$$

In dependences (3), (4): $x_{1}$ - molar concentration of glycerin in the mixture, $\% ; x_{2}$ - reaction duration of the reaction mixture, h.

The adequacy of the constructed mathematical model was checked using the Fisher criterion, which established the absence of a loss of consistency (the level of significance 
of the regression coefficients $p>0.05$ ). The value of the determination coefficient for the resulting model is 0.95093 (the value is close to unity). Thus, the mathematical model adequately describes the dependence of the concentration of potassium glycerate in the resulting product on the conditions of obtaining the product.

Table 1

Research planning matrix and experimental values of the response function

\begin{tabular}{|c|c|c|c|}
\hline $\begin{array}{c}\text { Experi- } \\
\text { ment No. }\end{array}$ & $\begin{array}{c}\text { Molar con- } \\
\text { centration of } \\
\text { glycerin in the } \\
\text { mixture, } \%\end{array}$ & $\begin{array}{c}\text { Duration of } \\
\text { reaction of } \\
\text { the reaction } \\
\text { mixture, } \mathrm{h}\end{array}$ & $\begin{array}{c}\text { Potassium glycer- } \\
\text { ate concentration } \\
\text { in the resulting } \\
\text { product, } \%\end{array}$ \\
\hline 1 & 50 & 2 & 31.25 \\
\hline 2 & 50 & 4 & 48.53 \\
\hline 3 & 50 & 6 & 58.60 \\
\hline 4 & 60 & 2 & 34.85 \\
\hline 5 & 60 & 4 & 75.77 \\
\hline 6 & 60 & 6 & 77.50 \\
\hline 7 & 70 & 2 & 45.78 \\
\hline 8 & 70 & 4 & 76.89 \\
\hline 9 & 70 & 6 & 79.54 \\
\hline
\end{tabular}

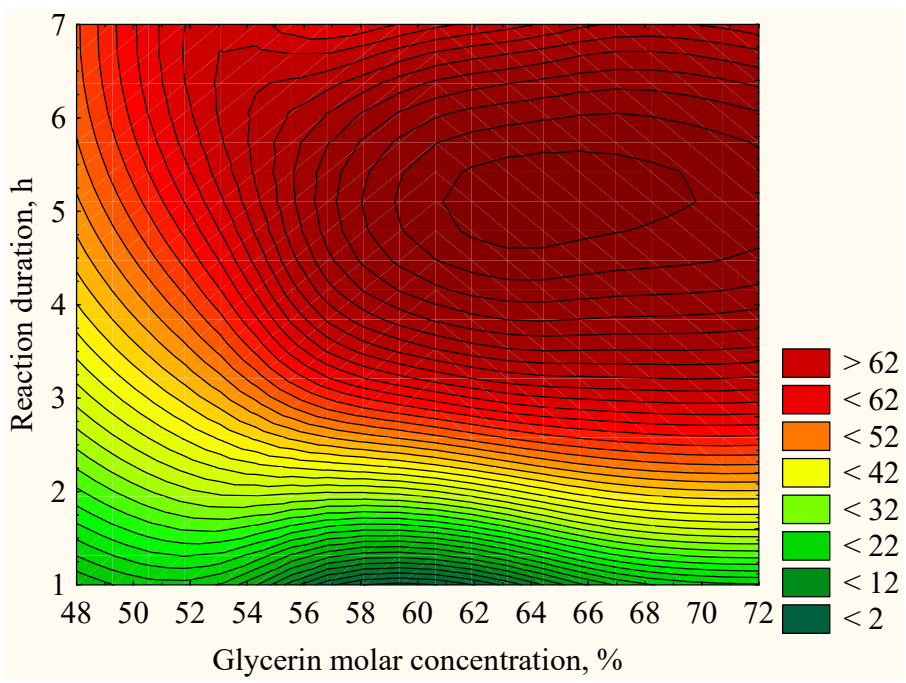

Fig. 2. Dependence of potassium glycerate concentration in the resulting product on the molar concentration of glycerin in the mixture and the duration of reaction of the reaction mixture

These data have been acquired regarding the basic substance concentration in the product. If we compare our results with those reported by other authors, in particular, with paper [7] that established that the molar ratio of glycerin to potassium hydroxide of 2:1 was rational, then one should note the effect of this indicator on the loss of free water mass during catalyst synthesis was studied. The determined rational temperature was $130^{\circ} \mathrm{C}$. However, water evaporation is not an indisputable indicator of the effectiveness of the catalyst synthesis process, since glycerin evaporates with water, and the concentration of glycerate in the mass remains unknown.

Current studies, specifically works [8-11], failed to consider the influence of conditions of obtaining on the quality of metal glycerates. And only the conditions for their further use, efficiency in various technological processes, in particular, regarding the production of biodiesel fuel, are shown.

\section{4. Determining the quality indicators of potassium} glycerate

The qualitative indicators of the obtained potassium glycerate were examined according to the procedures described in chapter 4.5. The melting point was $69^{\circ} \mathrm{C}$; the mass fraction of moisture, $0.8 \%$. Thus, based on the rational conditions, a quality product with a minimum moisture content has been obtained. values of the conditions for obtaining potassium glycerate represent the zone of rational values; further increase in parameters is impractical.

Thus, the rational values of the conditions for obtaining potassium glycerate are as follows: the molar concentration of glycerin in the mixture is $60 \%$; the duration of reaction of the components of the reaction mixture is 4 hours. Under these conditions, the concentration of potassium glycerate in the resulting product is $75.77 \%$.

\section{Discussion of results of studying the dependence of the effectiveness of obtaining potassium glycerate on the processing conditions of reagents}

The raw material for the synthesis of potassium glycerate (potassium hydroxide) has been studied. We have

Table 2 established the basic substance mass fraction of $85.5 \%$ and the mass fraction of potassium carbonate of $0.9 \%$.

Estimation values of potassium glycerate concentration in the resulting product, $\%$

\begin{tabular}{|c|c|c|c|c|c|c|c|c|c|}
\hline No. & 1 & 2 & 3 & 4 & 5 & 6 & 7 & 8 & 9 \\
\hline $\begin{array}{c}\text { Concentration of po- } \\
\text { tassium glycerate in the } \\
\text { resulting product, \% }\end{array}$ & 26.67 & 54.44 & 59.26 & 41.25 & 71.02 & 75.84 & 45.95 & 75.72 & 80.54 \\
\hline
\end{tabular}

The influence of the processing temperature of the components of the reaction mixture on the concentration of potassium glycerate in the resulting product has been determined (Fig. 1). 
It has been shown that an increase in temperature above $145{ }^{\circ} \mathrm{C}$ is impractical.

Based on Tables 1, 2, equation (4), and Fig. 2, the rational conditions for obtaining potassium glycerate were established: a molar concentration of glycerin in the mixture of $60 \%$, the duration of reaction of components of the reaction mixture of 4 hours. Under these conditions, the concentration of potassium glycerate in the resulting product was determined, which is $75.77 \%$. We have investigated the qualitative indicators of the obtained potassium glycerate: the melting point was $69^{\circ} \mathrm{C}$, the mass fraction of moisture was $0.8 \%$.

As regards available scientific data on the production of glycerate metals reported in [12-17], our work differs in that we have established the simultaneous effect of the basic conditions of production on the concentration of potassium glycerate in the resulting product. The appropriate rational conditions could make it possible to effectively implement the production of alkali metal glycerates in the manufacturing process, where there is a need for these substances. The obtained data on the concentrations of potassium glycerate in the product are essential when using glycerates as raw materials or catalysts in the production in various industries.

The formation of potassium glycerate occurs during the heating and evaporation of moisture from the reaction mass (a mixture of glycerin and an aqueous solution of potassium hydroxide). The presence of water leads to the decomposition of potassium glycerate, while glycerin and potassium hydroxide are again formed. Rational is to heat the mixture to a temperature of $145^{\circ} \mathrm{C}$.

With an increase in the duration of reaction of the reaction mixture and the molar concentration of glycerin in the mixture, the concentration of potassium glycerate in the resulting product increases. Increasing the duration of the process contributes to a more complete transformation of reagents into the resulting product. This work applied molar concentrations of glycerin in the mixture starting with $50 \%$ since lower concentrations of glycerin would contribute to the presence of potassium residue hydroxide in the resulting product. That may adversely affect the further processes of using glycerate, for example, leading to the saponification and loss of fats, while using potassium glycerate as a catalyst for the process of transesterification of oils and fats. In addition, glycerin solutions with water can evaporate during heating, which leads to loss of glycerin and incomplete conversion of potassium hydroxide. The results of our experiment showed that an increase in the molar concentration of glycerin in the mixture above $60 \%$, as well as the duration of reaction of the components of the reaction mixture for more than 4 hours, do not result in a significant increase in the value of the response function.

Our data on the rational conditions for the production of potassium glycerate could also be used to obtain glycerates of other alkaline metals (for example, sodium). It is necessary to take into consideration the actual concentrations of the basic substance in the hydroxides of alkaline metals, since during the storage of alkalis their partial transformation into carbonates occurs, as a result of which the real concentration of alkali in the reaction mass and the effectiveness of the process of obtaining glycerates decrease. The quality of glycerin also affects the efficiency of the process. Glycerin with a concentration of $99.5 \%$ was used in this work. In the case of apply- ing lower concentration glycerin, this fact should be taken into consideration when calculating the required number of reagents for the synthesis of potassium glycerate. It should be noted that the obtained data describe the process of synthesis of potassium glycerate under the conditions of the molar concentration of glycerin in the mixture from 50 to $70 \%$ and the duration of reaction from 2 to 6 hours. When using the values of these parameters outside the current study, it is necessary to perform additional research to establish the appropriate dependences, to check the adequacy of the mathematical model.

Promising areas of research into the production of metal glycerates are to study the physical and chemical properties of the obtained samples of glycerates, to investigate the temperature and thermal characteristics of phase transformations. Such data could make it possible to associate the purity of the resulting substance with the characteristics determined instrumentally. That would make it possible to quickly control the quality of the resulting metal glycerates, calculate the required amount for a particular technological process. The established rational conditions for obtaining potassium glycerate would make it possible to effectively use the resources of an enterprise since they represent the dependence of the concentration of the resulting product on various parameters. By varying the processing parameters of the mixture of reagents, high concentration glycerates could be obtained. Combining data on the concentration of glycerates and their physicochemical properties, it is possible to produce high-quality glycerates for different industry needs.

\section{Conclusions}

1. We have analyzed potassium hydroxide for its further use to produce potassium glycerate. The basic substance mass fraction has been determined, $85.5 \%$, as well as the mass fraction of carbonate potassium, $0.9 \%$. The value of the basic substance mass fraction in potassium hydroxide is used in the preparation of a solution to produce potassium glycerate.

2 . The influence of the heating temperature of the reaction mixture on the effectiveness of the production of potassium glycerate has been determined. The rational value of the heating temperature of the reaction mixture is $145^{\circ} \mathrm{C}$. Increasing the temperature above this value does not lead to a significant increase in the concentration of potassium glycerate in the resulting product.

3. The dependence of the concentration of potassium glycerate in the resulting product on the following conditions has been experimentally established: the molar concentration of glycerin in the reaction mixture and the duration of heating. Increasing the values of these indicators leads to an increase in the concentration of potassium glycerate. The rational values of conditions to produce potassium glycerate are as follows: a molar concentration of glycerin in the mixture is $60 \%$, the duration of reaction of components of the reaction mixture is 4 hours. Under these conditions, the concentration of potassium glycerate in the resulting product is $75.77 \%$.

4. The indicators of potassium glycerate obtained under the rational conditions have been determined: the melting point, $69{ }^{\circ} \mathrm{C}$; the mass fraction of moisture, $0.8 \%$. 


\section{References}

1. Rahmankulov, D. L., Kimsanov, B. H., Chanyshev, R. R. (2003). Fizicheskie i himicheskie svoystva glitserina. Moscow: Himiya, 200. Available at: https://www.studmed.ru/rahmankulov-dl-kimsanov-bh-chanyshev-rr-fizicheskie-i-himicheskie-svoystva-glicerina a990eae1973.html

2. Geibel, J. P., Kirchhoff, P. (2006). Pat. No. EP1976532A2. Fast acting inhibitor of gastric acid secretion. declareted: 27.01.2006; published: 11.11.2015. Available at: https://patents.google.com/patent/EP1976532A2/en

3. Novikov, O. O., Novykova, L. V., Semenchenko, O. O. (2004). Pat. No. 4382 UA. A method for the glass chemical treatment. No. 2004042569; declareted: 06.04.2004; published: 17.01.2005, Bul. No. 1. Available at: https://uapatents.com/2-4382-sposibkhimichno-obrobki-skla.html

4. Pradhan, S., Shen, J., Emami, S., Naik, S. N., Reaney, M. J. T. (2014). Fatty acid methyl esters production with glycerol metal alkoxide catalyst. European Journal of Lipid Science and Technology, 116 (11), 1590-1597. doi: https://doi.org/10.1002/ejlt.201300477

5. Hsiao, M.-C., Chang, L.-W., Hou, S.-S. (2019). Study of Solid Calcium Diglyceroxide for Biodiesel Production from Waste Cooking Oil Using a High Speed Homogenizer. Energies, 12 (17), 3205. doi: https://doi.org/10.3390/en12173205

6. Teslenko, A., Chernukha, A., Bezuglov, O., Bogatov, O., Kunitsa, E., Kalyna, V. et. al. (2019). Construction of an algorithm for building regions of questionable decisions for devices containing gases in a linear multidimensional space of hazardous factors. Eastern-European Journal of Enterprise Technologies, 5 (10 (101)), 42-49. doi: https://doi.org/10.15587/1729-4061.2019.181668

7. Pradhan, S., Shen, J., Emami, S., Mohanty, P., Naik, S. N., Dalai, A. K., Reaney, M. J. T. (2017). Synthesis of potassium glyceroxide catalyst for sustainable green fuel (biodiesel) production. Journal of Industrial and Engineering Chemistry, 46, $266-272$. doi: https://doi.org/10.1016/j.jiec.2016.10.038

8. Reyero, I., Arzamendi, G., Gandía, L. M. (2014). Heterogenization of the biodiesel synthesis catalysis: CaO and novel calcium compounds as transesterification catalysts. Chemical Engineering Research and Design, 92 (8), 1519-1530. doi: https:// doi.org/10.1016/j.cherd.2013.11.017

9. Lukić, I., Kesić, Ž., Zdujić, M., Skala, D. (2016). Calcium diglyceroxide synthesized by mechanochemical treatment, its characterization and application as catalyst for fatty acid methyl esters production. Fuel, 165, 159-165. doi: https://doi.org/ 10.1016/j.fuel.2015.10.063

10. Sánchez-Cantú, M., Reyes-Cruz, F. M., Rubio-Rosas, E., Pérez-Díaz, L. M., Ramírez, E., Valente, J. S. (2014). Direct synthesis of calcium diglyceroxide from hydrated lime and glycerol and its evaluation in the transesterification reaction. Fuel, 138, 126-133. doi: https://doi.org/10.1016/j.fuel.2014.08.006

11. Bradley, D., Levin, E., Rodriguez, C., Williard, P. G., Stanton, A., Socha, A. M. (2016). Equilibrium studies of canola oil transesterification using a sodium glyceroxide catalyst prepared from a biodiesel waste stream. Fuel Processing Technology, 146, 70-75. doi: https://doi.org/10.1016/j.fuproc.2016.02.009

12. Kovaliova, O., Tchoursinov, Y., Kalyna, V., Koshulko, V., Kunitsia, E., Chernukha, A. et. al. (2020). Identification of patterns in the production of a biologically-active component for food products. Eastern-European Journal of Enterprise Technologies, 2 (11 (104)), 61-68. doi: https://doi.org/10.15587/1729-4061.2020.200026

13. Sytnik, N., Kunitsa, E., Mazaeva, V., Chernukha, A., Bezuglov, O., Bogatov, O. et. al. (2020). Determination of the influence of natural antioxidant concentrations on the shelf life of sunflower oil. Eastern-European Journal of Enterprise Technologies, 4 (11 (106)), 55-62. doi: https://doi.org/10.15587/1729-4061.2020.209000

14. Papargyriou, D., Broumidis, E., de Vere-Tucker, M., Gavrielides, S., Hilditch, P., Irvine, J. T. S., Bonaccorso, A. D. (2019). Investigation of solid base catalysts for biodiesel production from fish oil. Renewable Energy, 139, 661-669. doi: https://doi.org/10.1016/ j.renene.2019.02.124 